\title{
SEROPREVALENCIA DEL MARCADOR ANTI CORE TOTAL DE HEPATITIS B EN EL HOSPITAL DANIEL ALCIDES CARRIÓN ESSALUd, TACNA
} 2,013-2017

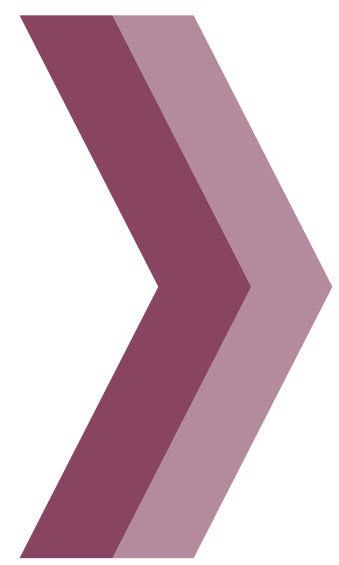

\author{
SEROPREVALENCE OF THE TOTAL \\ A NTI CORE MARKER OF \\ HEPATITIS B AT DANIEL ALCIDES \\ CARRIÓN HOSPITAL ESSALUd, \\ TACNA 2013-2017
}

\section{Malca Milla, Jaime Alberto}

1.Médico Patólogo Clínico - Hematólogo.

Médico asistente Hospital Daniel Alcides Carrión EsSalud Tacna

Docente de la Universidad Privada de Tacna.

\section{RESUMEN}

INTRODUCCIÓN: Una de las formas de la transmisión del virus de la hepatitis B (HBV) es a través de las transfusiones sanguíneas; por tal motivo, en el Perú, el tamizaje para despistaje del HVB forma parte de los marcadores serológicos obligatorios a realizar a los donantes de sangre. MATERIALES Y MÉTODOS: El presente estudio es de tipo retrospectivo, transversal, descriptivo. Se utilizó el registro de donantes de sangre del Hospital Daniel Alcides Carrión EsSalud- Tacna, de los años 2013 a 2017. Se seleccionó los que tuvieron marcador anticore total para Hepatitis B (anti HBCAg) reactivo. Luego, se construyó tablas de prevalencia de acuerdo a lugar de nacimiento y sexo del donante. RESULTADOS: Durante los años 2013 a 2017, hubieron 10296 donaciones de sangre. Se encontró 161 (1,56\%) donaciones con resultado anti $\mathrm{HBCAg}$ reactivo. La prevalencia más alta de reactividad se presentó en los donantes de sangre nacidos en la región Huánuco $(24,0 \%)$, Apurímac $(20,7 \%)$, Ucayali $(18,8 \%)$, Ayacucho $(16,7 \%)$ y Madre de Dios (13,3\%). En cuanto al sexo, 129 varones $(1,72 \%)$ de 7480 y 32 mujeres $(1,13 \%)$ de 2816 tuvieron marcador anti $\mathrm{HBCAg}$ reactivo, siendo significativa la asociación entre sexo y seroprevalencia $(p 0,32)$. CONCLUSIONES: La región de nacimiento del donante de sangre tiene importancia epidemiológica como valor predictivo de infección de hepatitis viral B. Existe asociación significativa entre la seroprevalencia del HBcAg y el sexo masculino.

Palabras claves: Donantes de sangre, hepatitis B, tamizaje. Perú (DeCS)

\section{ABSTRACT}

INTRODUCTION: one of the forms of transmission of hepatitis $B$ virus (HBV) is through blood transfusions; for this reason, in Peru, the screening test for HVB is part of the mandatory serological markers to be made to blood donors. MATERIALS AND METHODS: The present study is retrospective, transversal, descriptive. The blood donor registry of the Daniel Alcides Carrión EsSalud-Tacna Hospital from 2013 to 2017 was used. Those who had a total anti-core marker for Hepatitis B (anti $\mathrm{HBcAg}$ ) reactive were selected. Then, prevalence tables were constructed according to the donor's place of birth and sex. RESULTS: During the years 2013 to 2017, there were 10296 blood donations. 161 (1.56\%) donations with anti-HBCAg reactive result were found. The highest prevalence of reactivity occurred in blood donors born in the Huánuco region (24.0\%), Apurímac (20.7\%), Ucayali $(18.8 \%)$, Ayacucho (16.7\%) and Madre de Dios (13.3\%). Regarding sex, 129 males (1.72\%) of 7480 and 32 females $(1.13 \%)$ of 2816 had a reactive anti-HBcAg marker, being significant the association between sex and seroprevalence ( $p$ 0.32). CONCLUSIONS: The blood donor's region of birth has epidemiological importance as a predictive value of viral hepatitis $B$ infection. There is a significant association between the seroprevalence of $\mathrm{HBCAg}$ and the male sex.

Keywords: Blood donors, Hepatitis B, Screening, Peru (DeCS). 


\section{INTRODUCCIÓN}

El virus de la hepatitis B (HBV) puede transmitirse a través de las transfusiones de sangre y derivados sanguíneos, por tal motivo, el tamizaje para despistaje del HBV forma parte de los marcadores serológicos obligatorios a realizar en los donantes de sangre. Los marcadores serológicos que se realizan para el descarte de infección del virus de la hepatitis $B$ son el antígeno de superficie (HBsAg) y los anticuerpos totales contra el core del virus (anti $\mathrm{HBcAg}$ ). El Perú en general es considerado como uno de los países con endemicidad intermedia para la infección por el HBV (1)(2); a su vez, dentro del país existen diferencias de prevalencia entre las distintas regiones geográficas y políticas (1)(6), siendo mayor la prevalencia en la región geográfica de la selva y en los grupos étnicos nativos de la amazonía (1)(6).

Al realizar el tamizaje de las donaciones de sangre con las pruebas de ELISA, se pueden obtener resultados "reactivos" o "no reactivos"; un resultado "reactivo" indica una alta posibilidad de infección presente o pasada por alguno de los agentes etiológicos que se buscan con el tamizaje. Además, un resultado "reactivo" para cualquiera de los siete marcadores serológicos del tamizaje de donantes de sangre representa la pérdida de una unidad de sangre, asociada a las pérdidas económicas por costos de bolsas, tamizaje de marcadores y horas de trabajo. Asimismo, cabe la posibilidad de obtener resultados falsos negativos con efectos negativos sobre los receptores.

El Hospital Daniel Alcides Carrión del seguro social EsSalud (HDAC-Tacna), de la Red asistencial Tacna- Perú cuenta con un banco de sangre Tipo II, en donde se realiza la extracción, tamizaje y atención de las necesidades de sangre y derivados de los pacientes asegurados.

En el Banco de Sangre del HDAC -Tacna, se ha observado que la principal causa de pérdidas de unidades de sangre es la donación de sangre con marcador "reactivo" para anti HBcAg. Así, para el año 2004 se tuvo la prevalencia más alta de este marcador con un 3,39 \% de un total de 560 donantes, desde entonces se ha mejorado en la selección del donante y en el registro electrónico de la información, con ello, la prevalencia de este marcador ha disminuido a un $1,75 \%$ de 2283 donantes en el año 2017. A pesar de esta disminución, el marcador serológico anti HBcAg sigue siendo la principal causa de eliminación de unidades de sangre.

Los objetivos del presente estudio fueron determinar la seroprevalencia del marcador serológico anti $\mathrm{HBcAg}$ en los donantes del banco de sangre del HDAC - Tacna de los años 2013 a 2017, y determinar la prevalencia de este marcador respecto al lugar de nacimiento y sexo del donante.

\section{MATERIALES Y MÉTODOS}

El presente estudio es de tipo retrospectivo, transversal y descriptivo. Para su ejecución se buscó en el libro "Registro de Donantes de Sangre" del HDACTacna los donantes que tuvieron serología reactiva para el marcador anti HBcAg entre los años 2013 a 2017. Se registró el número de donante, y se procedió luego a la búsqueda de las fichas de donante para tomar los datos de lugar de nacimiento y sexo. Con esta información, se procedió a la construcción de tablas de prevalencia y al análisis estadístico de chi cuadrado para la variable sexo con valor de $p<0,05$.

En este banco de sangre, se realizó el tamizaje de las unidades durante los cinco años de este estudio en diferentes analizadores automatizados para pruebas de Elisa. Para el marcador anti $\mathrm{HBcAg}$, se utilizó pruebas de Elisa de tercera generación. En caso de pruebas reactivas, se realizó la repetición de la prueba para confirmar el resultado, concluyendo el proceso de tamizaje con este segundo análisis.

\section{RESULTADOS}

Entre los años 2013 a 2017, en el banco de sangre del HDAC -Tacna, se extrajo 10296 unidades de sangre, todas ellas fueron sometidas al tamizaje obligatorio, de acuerdo a la norma establecida en el Perú. 161 unidades $(1,56 \%)$ tuvieron serología reactiva para el marcador anti HBcAg. Como se puede apreciar en la tabla 1, hubieron donantes de todas las regiones políticas del Perú. Un total de 6217 donantes nacieron en la región Tacna, 4013 nacieron en otras regiones, principalmente en la región Puno, 66 donantes nacieron en otros países. Las tasas más altas de prevalencia del marcador anti HBcAg fueron para los donantes procedentes de las regiones políticas de: Huánuco, Apurímac, Ucayali, Ayacucho, Madre De Dios y Junín. A su vez, la tasa de prevalencia fue de 0,0\% para Amazonas, Cajamarca, Cerro de Pasco, Huancavelica, Ica, Lambayeque, Piura, y Tumbes. La cantidad de 7480 $(72,64 \%)$ donantes fueron de sexo masculino (Tabla 2$)$ 
la prevalencia para el sexo masculino fue mayor que para el sexo femenino.

\begin{tabular}{|c|c|c|c|}
\hline \multicolumn{4}{|c|}{$\begin{array}{l}\text { TABLA No } 1 \\
\text { Prevalencia del marcados anticore total para el virus de } \\
\text { la hepatitis B en donantes de sangre en relación al lugar } \\
\text { de nacimiento. } \\
\text { HOSPITAL DANIEL ALCIDES CARRIÓN ESSALUD TACNA } \\
\qquad 2013-2017\end{array}$} \\
\hline Región & Cantidad & Reactivos & Prevalencia \\
\hline AMAZONAS & 16 & 0 & 0.00 \\
\hline CAJAMARCA & 13 & 0 & 0.00 \\
\hline CERRO DE PASCO & 3 & 0 & 0.00 \\
\hline HUANCAVELICA & 4 & 0 & 0.00 \\
\hline ICA & 91 & 0 & 0.00 \\
\hline LAMBAYEQUE & 48 & 0 & 0.00 \\
\hline PIURA & 61 & 0 & 0.00 \\
\hline TUMBES & 5 & 0 & 0.00 \\
\hline TACNA & 6217 & 62 & 1.00 \\
\hline PUNO & 1085 & 15 & 1.38 \\
\hline LIMA & 607 & 10 & 1.65 \\
\hline MOQUEGUA & 997 & 16 & 1.60 \\
\hline AREQUIPA & 610 & 12 & 1.97 \\
\hline ANCASH & 43 & 1 & 2.33 \\
\hline EXTRANJERO & 66 & 3 & 4.55 \\
\hline LORETO & 37 & 2 & 5.41 \\
\hline SAN MARTíN & 18 & 1 & 5.56 \\
\hline CUsco & 174 & 10 & 5.75 \\
\hline LA LIBERTAD & 59 & 5 & 8.47 \\
\hline JUNIN & 39 & 4 & 10.26 \\
\hline MADRE DE DIOS & 15 & 2 & 13.33 \\
\hline AYACUCHO & 18 & 3 & 16.67 \\
\hline UCAYALI & 16 & 3 & 18.75 \\
\hline APURIMAC & 29 & 6 & 20.69 \\
\hline HUANUCO & 25 & 6 & 24.00 \\
\hline TOTAL & 10296 & 161 & 1.56 \\
\hline
\end{tabular}

\section{TABLA № 2}

Prevalencia del marcador anticore toral para el virus de la hepatitis $B$ en donantes de sangre en relación al sexo. HOSPITAL DANIEL ALCIDES CARRIÓN ESSALUD TACNA 2013-2017

\begin{tabular}{|cccc}
\hline Región & Donaciones & Casos & Prevalencia \\
\hline MASCULINO & 7480 & 129 & 1.72 \\
\hline FEMENINO & 2816 & 32 & 1.13 \\
TOTAL & 10296 & 161 & 1.56 \\
\hline
\end{tabular}

En 6 casos, se presentaron ambos marcadores de hepatitis B reactivos (anti HBcAg y antígeno de superficie), la positividad de ambos marcadores indica una infección persistente por el HBV. En 3 casos, se tuvo serología positiva para anti HBcAg y sífilis (Tabla 3 ).

TABLA No 3
Concomitancia del marcador anticore total para el virus
de la hepatitis B con otro marcadores en donantes de
sangre en relación al sexo.
HOSPITAL DANIEL ALCIDES CARRIÓN ESSALUD TACNA
2013-2017
MARCADOR
SOLO CORE
CORE +HBSAG
CORE+ HTLV
CORE +HVC
CORE+HIV
CORE + SÍFILIS
TOTAL
$\quad 148$

\section{DISCUSIÓN}

Se tuvo una prevalencia para el marcador anti $\mathrm{HBcAg}$ de 1,56\% -cifra menor a la reportada en dos estudios en donantes de sangre en el Perú-, uno de ellos en un banco de sangre de Lima 5,2\% (7), y otro en un banco de sangre del Callao (8) con 5,15\%. En otro estudio en la ciudad de Lima entre los años 2008 a 2013, en el Hospital Nacional Docente Madre Niño San Bartolomé, la prevalencia reportada fue de 4,63\% (9). Max Carlos Ramírez Soto reporta que un $35 \%$ de los donantes de sangre en un hospital de Apurímac Perú (10) tuvieron anti $\mathrm{HBcAg}$ reactivo. Así mismo, la prevalencia de nuestro estudio fue menor a la reportada en Argentina con un $2,01 \%$ (11), y Colombia con un $2,86 \%$ en un estudio nacional en el año 2006, y de $2,07 \%$ en el año 2010 (12).

El 60,4\% de los donantes del presente estudio nacieron en la región Tacna, siendo la prevalencia para esta región de 1,0\%. Este porcentaje disminuyó la prevalencia general del estudio. A partir de nuestro estudio, se puede inferir que la región Tacna tiene una endemicidad baja para el virus de la hepatitis $B$ de acuerdo al indicador anti $\mathrm{HBCAg}(13)$.

La baja prevalencia, se podría atribuir a la evaluación exhaustiva de los postulantes a donar sangre, así como a la mejora en la informatización de los registros de donaciones previas.

Hemos considerado utilizar la referencia de lugar de nacimiento, ya que la infección por el HVB pudo haberse producido a edades tempranas de la vida del 
donante nacido en zonas endémicas del virus (15) antes de migrar a la región Tacna.

En nuestro estudio podemos observar que los donantes de sangre nacidos en conocidas zonas endémicas de infección del HVB, tales como son Huánuco, Apurímac, Ucayali, Ayacucho y Madre de Dios tuvieron las más altas prevalencias para el marcador anti HBcAg (13)(14). La prevalencia hallada para los donantes nacidos en Loreto $(5,4 \%)$ y Amazonas $(0,0 \%)$ fue considerada baja a pesar de que estas zonas son valoradas como regiones endémicas (13)(14).

El año 1991, se incorporó la vacuna contra el HVB en la ciudad de Abancay (Apurímac), zona hiperendémica para el HVB. En el año 1994 se introdujo la vacuna en la ciudad de Huanta (Ayacucho). En el año 1996, se extendió la vacuna a los menores de 1 año en las zonas de alta y mediana endemicidad (13); por lo que la población protegida contra el HVB nacida en zonas endémicas debería tener cerca de 20 años de edad al tiempo en que se realizó esta investigación. La disminución en la incidencia de infección por el HVB producto de la inmunización por la vacuna podría observarse en años futuros.

Respecto al sexo del donante, el mayor porcentaje de donantes de sangre $(72,64 \%)$ fue de sexo masculino. Respecto a la prevalencia y el sexo, 129 (1,74\%) hombres y $32(1,13 \%)$ mujeres fueron reactivos para el anti HBcAg. Al análisis estadístico, se encontró asociación significativa entre el sexo masculino y la seropositividad del marcador ( $p 0,32$ ). En los estudios citados en la bibliografía se hizo referencia al mayor porcentaje de resultados reactivos en los donantes varones. Sin embargo, estas referencias citan el mayor porcentaje sobre el total de donantes reactivos, no tomando en cuenta que los donantes de sexo masculino son los donantes más frecuentes.

Podemos apreciar que, para la mayoría de los casos, se tuvo solo reactivo el marcador anti HBcAg lo que está asociado con infección pasada, probablemente recuperada. En seis casos, se tuvo concomitancia de anti $\mathrm{HBCAg}$ y antígeno de superficie propio de fase aguda o persistente de la hepatitis $B$.
La sífilis, el HTLV I/II, la hepatitis viral C y el virus de inmunodeficiencia humana comparten con el HBV mecanismos de transmisión, tales como las relaciones sexuales y las transfusiones de sangre. En nuestro estudio, se tuvo pocos casos de concomitancia de marcadores con otros agentes infecciosos.

Es necesario resaltar que al momento de la entrevista y evaluación médica del donante de sangre, se preguntó sobre el antecedente de haber sufrido hepatitis o haber estado en contacto íntimo con personas que sufrieron hepatitis. Asímismo, se realizó un examen médico general en el que, entre otros signos, se buscó ictericia. En consecuencia, los donantes aceptados para donar sangre no registraron historia pasada o presente de hepatitis. De aquí, podemos inferir que este porcentaje de la población que tuvo reactivo el marcador anti HBcAg cursó con una infección subclínica, lo que contribuye a la persistencia de la enfermedad en la población.

Nuestro estudio tuvo la limitación de no contar con mayor precisión sobre la ciudad de nacimiento del donante, ya que una región política engloba ciudades ubicadas en diferentes regiones geográficas. Asimismo, se tuvo la limitante de la confirmación del resultado reactivo, por cuanto se ha observado que hasta un $18,8 \%$ de los casos de anti HBcAg reactivo corresponden a falsos positivos con las pruebas de Elisa (12).

En conclusión, se tiene una prevalencia de 1,56\% para el marcador anti HBcAg en los donantes de sangre del HDAC-Tacna EsSalud entre los años 2013 a 2017. La prevalencia más alta fue para los donantes nacidos en zonas endémicas como Huánuco, Apurímac, Ucayali, Ayacucho, y Madre de Dios. Se encontró asociación significativa entre el sexo y la seropositividad para el marcador anti $\mathrm{HBcAg}$.

Se recomienda utilizar pruebas rápidas para anti $\mathrm{HBcAg}$ como screening en postulantes a donar sangre nacidos en zonas endémicas que tengan más de 20 años de edad. 


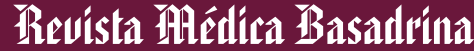

\section{REFERENCIAS BIBLIOGRÁFICAS}

1. Méndez $M$, Arce $M$, Kruger $H$, Sánchez $S$. Prevalencia de marcadores serológicos de hepatitis vírica en diversos grupos de población del Perú. Bol Oficina Sanit Panam. 1989;106(2):12738.

2. Vildósola H, Farfán G, Colan E, Delgado G, Mendoza L, Pineda $\mathrm{R}$, et al. Prevalencia del antígeno de superficie del virus de hepatitis B en población general de costa sierra y selva del Perú. Reporte preliminar. Rev Gatroenterol Perú. 1990; 10(3)96-101.

3. Cabezas C, Suarez M, Romero G, Carrillo C, García MP, Reátegui J, et al. Hiperendemicidad de hepatitis viral B y delta en pueblos indígenas de la Amazonía Peruana. Rev Perú Med Exp Salud Paública. 2006; 23(2): 114-22.

4. Morales J, Fuentes- Rivera J, Delgado-Silva C,Matta-Solís H.Marcadores de infección para hepatitis viral en donantes de sangre de un hospital nacional de Lima metropolitana. Rev Peru Med Exp Salud Publica . 2017;34(3):466-71.

5. Alvarez L, Tejada-Llacsa P, Melgarejo-García G, et al. Prevalencia de hepatitis $B$ y $C$ en el Banco de sangre de un hospital en Callao,Perú. Rev Gastroenterol Perú. 2017; 37(4):346-9.

6. Moya J, Julcamanyan E. Seroprevalencia de marcadores infecciosos causantes de pérdidas de hemodonaciones en el servicio de Banco de Sangre del Hospital Nacional Docente Madre Niño San Bartolomé de enero 2008 a diciembre del 2013. Horiz Med. 2014;14(4):6-14.

7. Ramírez -Soto MC, Huichi-Atamari M. Hepatitis B en donantes de sangre de un hospital en Apurímac,Perú. Rev Perú Med Exp Salud Pública. 2012;29(1):163-4

8. Fichman DM, Blejer JL, Liveralla BI, Re VE, Bartoli S, Bustos JA, et al. Prevalence and trends of markers odf hepatitis $B$ virus, hepatitis $\mathrm{C}$ virus and human deficiency virus in Argentina blood donors. BMC Infect Dis. 2014;14 (218):1-9.

9. Beltrán-Duran $M$, Berrio-Pérez $M$, Bermúdez-Forero $M$, et al. Perfiles serológicos de hepatitis $B$ en donantes de sangre con anti-HBc reactivos. Rev salud pública. 2014;16 (6): 847-858.

10. Cabezas C. Hepatitis virales B y Delta: epidemiología y prevención en el Perú. Rev Perú Med Exp Salud Pública. 2002;19(3):150-61.

11. Cabezas C. Hepatitis viral B y delta en el Perú: epidemiología y bases para su control. Rev Peru Med exp salud pública. 2007; 24 (4) : 378-97.

12. Segovia G, Galvan K, Garcia V, Huamaní L, Gotuzzo E. Prevalencia de marcadores serológicos de hepatitis $B$ y delta e infección intrafamiliar en el valle del río Pampas. P

\section{Correspondencia}

Jaime Alberto Malca Milla

E-mail: jmmmap@hotmail.com
Fecha de recepción: 10 de mayo de 2018 Fecha de aceptación: 10 de junio de 2018 